Open Access

\title{
Late infantile metachromatic
} leukodystrophy: Clinical manifestations of five Taiwanese patients and Genetic features in Asia

\author{
Hsiang-Ru Liaw ${ }^{2+}$, Hsiu-Fen Lee ${ }^{2,3+}$, Ching-Shiang Chi ${ }^{1,3^{*}}$ and Chi-Ren Tsai ${ }^{2,4}$
}

\begin{abstract}
Background: This study was conducted to describe the clinical and genetic features of patients with late infantile metachromatic leukodystrophy.

Methods: Clinical and genetic manifestations of five Taiwanese patients with late infantile metachromatic leukodystrophy from January 2003 to April 2014 were reviewed. The genetic features of such patients reported in Asian countries during a period of 20 years were also analyzed.

Results: The median age at disease onset was 1 year and 3 months with the first clinical symptom being gait disturbance. All five patients became bed-ridden at a median age of 2 years and 5 months. Nerve conduction velocity revealed demyelinating polyneuropathy and brain MRI disclosed tigroid and leopard skin pattern of dysmyelination in all 5 patients. All patients had decreased ARSA activities in leukocytes accounting for $15.88 \%$ to $30.75 \%$ of controls. Five novel mutations, p.A316D, p.G303R, p.Q176X, p.R293X, and c.749 insGCGGGCCA, were identified in our case series. Eighteen patients, including our 5 patients, were reported in Asian countries. A total of 22 different disease-causing alleles were found, in which p.W320X was identified in Taiwan and China, and p.G101V was found in Taiwan and Korea.

Conclusions: Patients with late infantile metachromatic leukodystrophy exhibited a rapid and devastating clinical course. The pattern of dysmyelination on brain MRI together with peripheral demyelination polyneuropathy indicates that evaluation of ARSA activity in leukocytes is warranted. A wide diversity of ARSA gene mutations was noted in Asia.
\end{abstract}

Keywords: Metachromatic leukodystrophy, ARSA gene mutation, Taiwan, Asia

\section{Background}

Metachromatic leukodystrophy (MLD) is a rare autosomal recessive inherited disease, which is caused by a deficiency in the enzyme activity of Arylsulfatase A (ARSA). ARSA is required for the hydrolysis of sulfated glycosphingolipids, which are also known as sulfatides, and its deficiency results in excessive accumulation of sulfatide in myelin in the nervous system, the bile ducts of the liver, and the distal tubules of the kidney [1-3].

\footnotetext{
* Correspondence: chi-cs@hotmail.com

${ }^{\dagger}$ Equal contributors

'Department of Pediatrics, Tungs' Taichung Metroharbor Hospital, 699,

Taiwan Boulevard Sec. 8, Wuchi, Taichung 435, Taiwan

${ }^{3}$ School of Medicine, Chung Shan Medical University, 110, Sec. 1, Jianguo N.

Rd, Taichung 40201, Taiwan

Full list of author information is available at the end of the article
}

ARSA deficiency results from a mutation in the ARSA gene, which spans $3.2 \mathrm{~kb}$ of genomic DNA on chromosome 22q13 [2, 4]. Clinically, the differences in residual enzyme activity of ARSA cause a great diversity of onset age of the disease and the severity of the clinical course. Based on the age of disease onset, MLD can be divided into three forms: late infantile, juvenile, and adult MLD [4].

Late infantile MLD is the most common form of MLD, which accounts for 50-60\% of all cases [5] and incidence is estimated to range from 1 in 40,000 to 1 in 170,000 newborns [6]. The age of disease onset is usually between 18 and 24 months with the first recognizable feature of gait disturbance. Afterwards, rapid deterioration of motor, speech, and intellectual functions 
develop over the following months [7, 8]. Because of the profound ARSA deficiency and progressive demyelination, such cases usually manifest a rapid and devastating neurodegenerative clinical course [1]. Inevitable neurological sequelae develop as the disease progresses, such as decorticate postures, impaired feeding and swallowing due to pseudobulbar palsies, seizures, and severe psychomotor retardation. Those patients eventually expire within the first decade of life [4]. Currently, feasible therapeutic options are limited to palliative and supportive treatments [1, 2, 9-12].

Here we report the clinical manifestations, neuroimaging studies, ARSA enzyme activity, ARSA gene mutations, and neurological outcomes of five Taiwanese patients with late infantile MLD. In addition, the differences in ARSA gene mutations between Taiwan and other Asian countries are noted.

\section{Methods}

\section{Study sample}

This was a retrospective, uncontrolled, nonblinded study in which cases from a period spanning over a decade were analyzed. Between January 2003 and April 2014, a total of five patients diagnosed with late infantile MLD were enrolled. All patients underwent detailed physical and neurological examinations, and basic laboratory tests, including complete blood count, blood sugar, creatine phosphokinase, electrolytes, liver function test, renal function test, blood lactate level, arterial blood gas analysis, and urine routine, as well as metabolic surveys, including assays of blood amino acids and urinary organic acids. Spinal tap was administered to patients with their parents' consent, and the values of cerebrospinal fluid (CSF) and assay of CSF amino acids were determined. Neurophysiological studies, including electroencephalography (EEG), auditory evoked potential (AEP), and visual evoked potential (VEP) were performed. Biochemical enzyme activities for lysosomal disorders were analyzed. Brain magnetic resonance imaging (MRI) and nerve conduction velocity (NCV) were performed with their parents' consent.

The diagnostic criteria of late infantile MLD in our case series included: 1) disease onset at age younger than 2 years following by a clinically devastating regression of motor, language, and cognition functions; 2) tigroid and leopard skin pattern of dysmyelination on the brain MRI; 3) decreased ARSA activity in leukocytes; and 4) pathognomonic ARSA gene mutations [4].

\section{Molecular analysis}

Genomic DNA was extracted from peripheral blood samples. All the exons of the ARSA gene were amplified by polymerase chain reaction (PCR) with their corresponding intronic primers. PCR products were subjected to bidirectional sequencing using a Big-Dye Terminator v3.1 Cycle Sequencing Kit and an ABI Prism 3100 genetic analyzer (Applied Biosystems, Foster City, CA). The protein sequence was based on GenBank accession no. NM_00487.5. Written informed consent was obtained from all participants' parents prior to collection of blood samples for molecular studies.

\section{Data collection}

The medical records of these five patients were reviewed. Patients' clinical information, including gender, age at disease onset, histories of developmental milestones prior to the onset of the disease, duration between disease onset and psychomotor regression, and clinical outcomes, were collected. Laboratory data, neuroimaging findings, neurophysiological studies, ARSA enzyme activities, and ARSA gene mutations were analyzed. This study was approved by the Institutional Review Board (IRB TCVGH No. CF14213).

\section{Literature review}

We searched the MEDLINE and PubMed database using the keywords "metachromatic leukodystrophy". We reviewed cases diagnosed with late infantile MLD and reported in Asian countries from the period 1995 to 2014. In our study, patients with molecular analysis of ARSA gene mutations were recruited. In order to make meaningful comparisons between ARSA gene mutations in Taiwan and those reported in other Asian countries, the genomic data of all patients identified in the MEDLINE and PubMed search were re-interpreted with respect to the change in protein sequences of ARSA amino acids based on GenBank accession no. NM_00487.5 if a patient's sequencing results were originally interpreted by GenBank accession no. NM_00487.3 or GenBank accession no. NM_00487.4.

\section{Results}

The clinical features, laboratory data, neurophysiological studies, and neuroimaging findings of the five late infantile MLD Taiwanese patients, three boys and two girls, are shown in Table 1.

The median age at disease onset was 1 year and 3 months, with a range from 1 year 2 months to 1 year and 11 months. All of the patients exhibited the first recognizable clinical symptoms of gait disturbances with brisk deep tendon reflexes and increased tonicity over the bilateral lower legs upon neurological examinations at the first visit. After the onset of the disease, rapid psychomotor regression developed. All patients became bedridden at a median age of 2 years and 5 months, ranging from 1 year 7 months to 3 years and 
Table 1 Clinical manifestations, laboratory data, neurophysiological studies, and neuroimaging findings of five patients with late-infantile metachromatic leukodystrophy

\begin{tabular}{|c|c|c|c|c|c|}
\hline Patient & 1 & 2 & 3 & 4 & 5 \\
\hline Gender & M & $\mathrm{F}$ & $\mathrm{F}$ & M & M \\
\hline Age at disease onset & $1 \mathrm{yr} 3 \mathrm{mo}$ & 1 yr $2 \mathrm{mo}$ & $1 \mathrm{yr} 3 \mathrm{mo}$ & $1 \mathrm{yr} 2 \mathrm{mo}$ & $1 \mathrm{yr} 11 \mathrm{mo}$ \\
\hline Age at first visit & $2 \mathrm{yr} 4 \mathrm{mo}$ & $1 \mathrm{yr} 11 \mathrm{mo}$ & $2 \mathrm{yr}$ & 1 yr $8 \mathrm{mo}$ & $2 \mathrm{yr}$ \\
\hline \multicolumn{6}{|l|}{ Neurological examinations at first visit } \\
\hline Muscle tone & Hypertonicity & Hypertonicity & Hypertonicity & Hypertonicity & Hypertonicity \\
\hline Deep tendon reflexes & Increased & Increased & Increased & Increased & Increased \\
\hline Babinski sign & Negative & Positive & Positive & Positive & Positive \\
\hline Ankle clonus & Negative & Negative & Positive & Negative & Positive \\
\hline Posturing & Spasticity & Spasticity & Decorticate & Spasticity & Spasticity \\
\hline \multicolumn{6}{|l|}{ Laboratory data } \\
\hline $\begin{array}{l}\text { CSF protein level, mg/dl } \\
\text { (Normal range, } 20-45 \mathrm{mg} / \mathrm{dl} \text { ) }\end{array}$ & Not done & 135 & 200 & 171.4 & Not done \\
\hline \multicolumn{6}{|l|}{ Neurophysiological studies } \\
\hline \multicolumn{6}{|l|}{ Electroencephalography } \\
\hline At first visit & AS & AS & AS & AS & AS \\
\hline Follow-up & Not done & AS & $A S, B S$ & BS, focal spikes & BS \\
\hline Auditory evoked potential & No response & Normal & Normal & No response & Not done \\
\hline Visual evoked potential & Right side delay & Normal & Normal & Normal & Not done \\
\hline \multicolumn{6}{|l|}{ Nerve conduction velocity } \\
\hline Demyelinating polyneuropathy & Yes & Yes & Yes & Yes & Yes \\
\hline \multicolumn{6}{|l|}{ Neuroimaging findings } \\
\hline \multicolumn{6}{|l|}{ Brain MRI } \\
\hline Dysmyelination pattern resembling tiger skin & Yes & Yes & Yes & Yes & Yes \\
\hline \multicolumn{6}{|l|}{ Spinal MRI } \\
\hline Signal change over the white matter & Not done & Yes & Yes & Not done & Not done \\
\hline \multicolumn{6}{|l|}{ Psychomotor regression } \\
\hline Bed-ridden status, age & $2 \mathrm{yr} 5 \mathrm{mo}$ & $2 \mathrm{yr} 2 \mathrm{mo}$ & 3 yr $4 \mathrm{mo}$ & $1 \mathrm{yr} 7 \mathrm{mo}$ & 3 yr $6 \mathrm{mo}$ \\
\hline Being unable to speak, age & Not available & $2 \mathrm{yr}$ & $3 \mathrm{yr}$ & $1 \mathrm{yr} 7 \mathrm{mo}$ & $3 \mathrm{yr}$ \\
\hline Loss of eye contact, age & Not available & Not available & 3 yr $5 \mathrm{mo}$ & 2 yr $9 \mathrm{mo}$ & $4 \mathrm{yr} 4 \mathrm{mo}$ \\
\hline Seizure onset, age & Not available & Not available & $3 \mathrm{yr}$ & Never & 3 yr $6 \mathrm{mo}$ \\
\hline \multicolumn{6}{|l|}{ Neurological follow-up } \\
\hline Gastric tube implantation for feeding, age & Not available & 2 yr $6 \mathrm{mo}$ & 3 yr $8 \mathrm{mo}$ & $2 \mathrm{yr}$ & 3 yr $6 \mathrm{mo}$ \\
\hline Home BiPAP for respiratory support, age & Not available & $5 \mathrm{yr} 1 \mathrm{mo}$ & Nil & Nil & Nil \\
\hline \multirow[t]{2}{*}{ Outcome } & Loss of follow-up & Died & Loss of follow-up & Died & Alive \\
\hline & & $7 \mathrm{yr} 4 \mathrm{mo}$ & & 4 yr $9 \mathrm{mo}$ & $8 \mathrm{yr}$ \\
\hline
\end{tabular}

$A S$ absence of sleep spindles, $B S$ background slowing, CSF cerebrospinal fluid, $F$ female, $M$ male, mo months, $M R I$ magnetic resonance imaging, $y r$ years

6 months. The times at which language function regressed to "unable to speak" and social function deteriorated to "loss of eye contact" were available in 4 and 3 patients, respectively. The median age of symptoms in the aforementioned patients was 2 years and 6 months and 3 years and 5 months, respectively. Epileptic seizures occurred in patients 3 and 5 at age 3 years and 3 years and 6 months, respectively. Regarding the clinical outcomes during the long-term period of follow-up, patients 2 and 4 died of respiratory failure at the ages of 7 years and 4 months and 4 years and 9 months, respectively. Patient 5, aged 8 years, is still alive with significant neurological sequelae. Patients 1 and 3 were lost to follow-up.

Three patients received a spinal tap and all of them showed elevated levels of protein in CSF analysis. The results of neurophysiological studies revealed that patients 1 and 4 showed no response with respect to AEPs 
and patient 1 had a delayed response on the VEP evaluation. EEG at the first visit revealed nonspecific findings with absence of sleep spindles in all five patients. The follow-up EEGs were recorded depending on the patients' clinical condition: EEGs showed nonspecific findings with background slowing in three patients and focal spikes in patient 4. NCV and brain MRI were performed in all five patients. NCVs revealed demyelinating polyneuropathy. Cranial MRIs disclosed tigroid and leopard skin pattern of dysmyelination (Fig. 1a-o). Spinal MRIs of patients 2 and 3 showed signal changes over the white matter.

ARSA activity in leukocytes and ARSA gene mutations in our case series are shown in Table 2. All cases had significantly decreased ARSA activities in leukocytes with levels of 11.29 to $21.86 \mathrm{nmol} /$ per milligram of protein/hr (normal, > $71 \mathrm{nmol} /$ per milligram of protein/hr), which accounted for $15.88-30.75 \%$ of controls. For the ARSA gene mutations, a total of eleven alleles were identified, including an additional pseudodeficiency allele of p.N352S in patient 2 (Fig. 2a-e). There were nine different diseasecausing alleles, including four missense mutations of p.A316D, p.F249S, p.G101V, and p.G303R, three nonsense mutations of p.W320X, p.Q176X, and p.R293X, and two frameshift mutations of c.1344_1345 dupC and c.749 insGCGGGCCA. Five alleles, p.A316D, p.G303R, p.Q176X, p.R293X, and c.749 insGCGGGCCA, were novel mutations. All our reported cases were heterozygous gene mutations.

As shown in Table 2, 13 patients with late infantile MLD in Asian countries were reported in the period 1995-2014 [13-19], including seven from India, three from Japan, two from China, and one from Korea. Based on GenBank accession no. NM_00487.5 for changes in protein sequences of ARSA amino acids, a wide diversity of ARSA gene mutations were found. Excluding 3 cases in India for whom 3 alleles were not found, 15 different alleles in 23 alleles were identified. Six of 13 patients carried heterozygous gene mutations and seven were homozygous ARSA gene mutations. Taken together, there was a total of 18 patients, including our 5 patients, diagnosed with late infantile MLD with ARSA gene mutations in Asia, and 22 different alleles were identified. p.W320X was found in Taiwan and China, and p.G101V in Taiwan and Korea. Both p.W320X and p.G101Vwere found in three of the 33 alleles, with allele frequency of $9 \%$.

\section{Discussion}

The clinical course of patients with late infantile MLD typically involves initial presentation of unsteady gait at the age of 1 year and 6 months, according to reports in the literature. Around the age of 3 years, they no longer have any locomotion activity and head or trunk control is absent, ultimately leading to bed-ridden status $[7,20]$. In addition to motor function regression, they also experience the first language difficulties at a median age of about 2.5 years, and loss of verbal communication occurs at about 2 to 2.5 years after the onset of the disease [8]. Overall, our case series also observed a similar clinical course to those reported in the literature report. The median durations between disease onset and motor function regression to bed-ridden status and no verbal communication were 1 year and 2 months, and 11.5 months, respectively. Regarding the clinical outcomes, there are no reliable treatment options for patients with late infantile MLD [1,9-12]. With supportive treatment, including gastric tube placement for feeding and antibiotic coverage during infection, patients could survive in a vegetative state for years [20], but they often die of aspiration pneumonia or bronchopneumonia within the first decade of life [4]. Patients 2 and 4 in our series died of respiratory failure 6 years and 2 months and 3 years and seven months, respectively, after disease onset.

In patients with late infantile MLD, T2-weighted brain MRI usually showed symmetric high signal intensity initially in the parietal-occipital central white matter, followed by frontal central white matter changes, which then spread to the commissural fibers of the corpus callosum and the periventricular white matter with a homogeneous change of pattern parallel to the course of the disease [21-24]. In addition to central nervous system (CNS) involvement, peripheral neuropathy was also evident in these patients, which was associated with uniform slowing of both motor and sensory NCVs and risk of developing unsteady gait and ataxia [25-28]. All of our 5 patients had characteristic tigroid and leopard skin pattern of dysmyelination on brain MRI [29] and demyelination polyneuropathy on NCV. If there appears to be involvement in both the CNS and peripheral nerve system in patients with suspected late infantile MLD, this could provide a valuable diagnostic clue for clinical pediatric neurologists to proceed to the next step and evaluate ARSA activity in leukocytes [30].

A pathogenic factor of late infantile MLD is absence or dramatic loss of ARSA activity, which usually accounts for less than $15 \%$ of controls [31]. Our case series showed the reduction in ARSA activity ranged from $15.88-30.75 \%$ compared with controls. However, low ARSA level was not sufficient to make a definitive diagnosis of MLD. In healthy individuals, some people have ARSA activity as low as $15-50 \%$ of controls. This condition is called ARSA pseudodeficiency, which is a polymorphism [31]. Although a slight deficiency in ARSA activity, with levels 50-70 \% that of controls, might be associated with pervasive developmental disorders, ARSA pseudodeficiency does not lead to neurological impairments [31]. In the general population, the frequency of ARSA pseudodeficiency alleles is about 7$15 \%$. Therefore, an accurate diagnosis of late infantile 

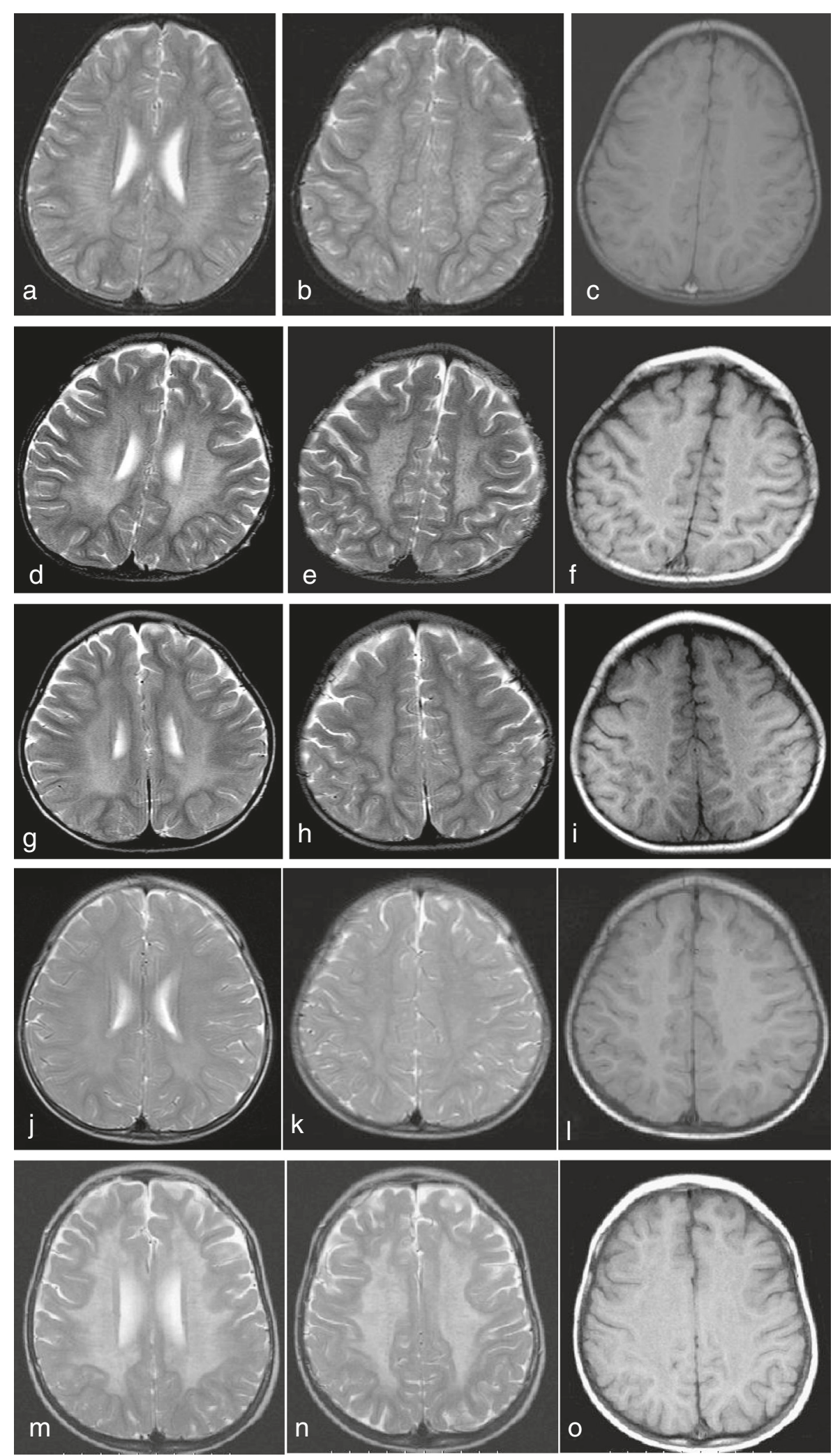

Fig. 1 (See legend on next page.) 
(See figure on previous page.)

Fig. 1 Cranial MRIs of our patients. a-c represents patient 1, d-f patient 2, g-i patient 3, j-1 patient 4, and $\mathbf{m}$-o patient 5. (a, d, g, j, m) Hypointense radially oriented stripes and dots seen within the hyperintense cerebral white matter (resembling tiger skin) on T2-weighted axial imaging. (b,e, $\mathbf{h}, \mathbf{k}, \mathbf{n})$ Hypointense dots resembling leopard skin seen on T2-weighted axial imaging at the level of centrum ovale. $(\mathbf{c}, \mathbf{f}, \mathbf{i}, \mathbf{l}, \mathbf{o})$ Iso to hyperintense dots seen in the cerebral white matter on T1-weighted imaging. This pattern of dysmyelination resembles the skin of tiger (radial stripes) and leopard (dots), the so-called tigroid and leopard pattern of dysmyelination in metachromatic leukodystrophy

MLD is based on a combination of clinical features, biochemical analysis of low residual enzyme activity of ARSA in leukocytes, together with pathognomonic ARSA gene mutations [32]. Regarding the correlation between ARSA activity and age of disease onset, we observed that our patients 1 to 4 had lower ARSA activities, ranged from 11.29 to $15.23 \mathrm{nmol} / \mathrm{mg}$ Protein/ $\mathrm{hr}$, whose disease onset were between 1 year 2 months old and 1 year 3 months old. Patient 5 had ARSA activity of $21.86 \mathrm{nmol} / \mathrm{mg}$ Protein/hr, and his disease onset was 1 year 11 months old. It seemed that lower residual enzyme activities were associated with an earlier onset. However, in India report, patient 9 with undetectable ARSA activity had disease onset at 2 years 3 months old. Our limitation was that the case numbers were too small to draw a definitive conclusion.

Table 2 ARSA enzyme activities and ARSA gene mutations in our case series and in reported cases in other Asian countries

\begin{tabular}{|c|c|c|c|c|c|c|}
\hline \multirow{2}{*}{$\begin{array}{l}\text { Patient } \\
\text { [Ref] }\end{array}$} & \multirow{2}{*}{$\begin{array}{l}\text { Age of } d z \\
\text { onset }\end{array}$} & \multirow{2}{*}{$\begin{array}{l}\text { ARSA enzyme } \\
\text { activity, nmol/mg } \\
\text { Protein/hr } \\
\text { (Normal reference) }\end{array}$} & \multirow{2}{*}{$\begin{array}{l}\text { ARSA } \\
\text { enzyme } \\
\text { activity, } \\
\% \text { of control } \\
\text { range }\end{array}$} & \multicolumn{3}{|c|}{ ARSA gene mutation Protein sequence of ARSA amino acid change by different GenBank } \\
\hline & & & & $\begin{array}{l}\text { GenBank accession } \\
\text { no. NM_00487.3 }\end{array}$ & $\begin{array}{l}\text { GenBank accession } \\
\text { no. NM_00487.4 }\end{array}$ & $\begin{array}{l}\text { GenBank accession no. } \\
\text { NM_00487.5 }\end{array}$ \\
\hline \multicolumn{7}{|l|}{ Taiwan } \\
\hline 1 & $1 \mathrm{yr} 3 \mathrm{mo}$ & $11.30(>71.1)$ & 15.89 & Not used & Not used & p.A316D a/p.W320X \\
\hline 2 & $1 \mathrm{yr} 2 \mathrm{mo}$ & $11.81(>71.1)$ & 16.61 & Not used & Not used & $\begin{array}{l}\text { p.F249S/c.1344_1345 dupC } \\
\text { p.N352S b }\end{array}$ \\
\hline 3 & $1 \mathrm{yr} 3 \mathrm{mo}$ & $11.29(>71.1)$ & 15.88 & Not used & Not used & p.Q176X a /p.R293X a \\
\hline 4 & $1 \mathrm{yr} 2 \mathrm{mo}$ & $15.23(>71.1)$ & 21.42 & Not used & Not used & p.G101V/c.749 insGCGGGCCA a \\
\hline 5 & $1 \mathrm{yr} 11 \mathrm{mo}$ & $21.86(>71.1)$ & 30.75 & Not used & Not used & p.G101V/p.G303R a \\
\hline \multicolumn{7}{|l|}{ India } \\
\hline 6 [13] & $1 \mathrm{yr} 6 \mathrm{mo}$ & $1.23(>50)$ & 2.46 & Not used & $c .459+1 G>A /$ not found & c. $465+1 G>A /$ not found \\
\hline $7[13]$ & $2 \mathrm{yr} 4 \mathrm{mo}$ & $2.43(>50)$ & 4.86 & Not used & p.Y33S/not found & p.Y35S/not found \\
\hline 8 [13] & $1 \mathrm{yr} 6 \mathrm{mo}$ & $2.45(>50)$ & 4.90 & Not used & p.R311Q/p.R311Q & p.R313Q/ p.R313Q \\
\hline 9 [13] & $2 \mathrm{yr} 3 \mathrm{mo}$ & Undetectable $(>50)$ & NA & Not used & $\begin{array}{l}c .459+1 G>A / c .459+ \\
1 G>A\end{array}$ & $c .465+1 G>A / c .465+1 G>A$ \\
\hline 10 [13] & $1 \mathrm{yr} 6 \mathrm{mo}$ & $0.83(>50)$ & 1.66 & Not used & $\begin{array}{l}\text { c. } 752 \_753 i n s T / \text { not found } \\
\text { c. } 1524+95 \mathrm{~A}>\mathrm{G} \text { b }\end{array}$ & $\begin{array}{l}\text { c.758_759insT/not found } \\
\text { c. } 1530+95 \mathrm{~A}>\mathrm{G} \text { b }\end{array}$ \\
\hline $11[13]$ & $2 \mathrm{yr} 6 \mathrm{mo}$ & $1.40(>50)$ & 2.80 & Not used & p.R390W/ p.R390W & p.R392W/ p.R392W \\
\hline 12 [13] & $2 \mathrm{yr}$ & $5.00(>50)$ & 10.0 & Not used & p.G245R/ p.G245R & p.G247R/ p.G247R \\
\hline \multicolumn{7}{|l|}{ Japan } \\
\hline $13[14]$ & NA & NA & NA & Unknown & Unknown & p.Q155H/ p.G310V \\
\hline 14 [15] & NA & NA & NA & Unknown & Unknown & p.L300S/ c. $225+2 \mathrm{~A}>\mathrm{G}$ \\
\hline 15 [16] & $1 \mathrm{yr} 11 \mathrm{mo}$ & 15.80 (109.0-217.2) & 14.50 & Not used & Not used & p.P138T/ p.P138T \\
\hline \multicolumn{7}{|l|}{ China } \\
\hline 16 [17] & $1 \mathrm{yr} 5 \mathrm{mo}$ & $7.00(38.9-98.3)$ & 17.99 & p.W318X/ p.W318X & Not used & p.W320X/ p.W320X \\
\hline 17 [18] & $1 \mathrm{yr} 7 \mathrm{mo}$ & 1.19 (NA) & NA & Not used & Not used & c.622delC/ c.622delC \\
\hline \multicolumn{7}{|l|}{ Korea } \\
\hline 18 [19] & $1 \mathrm{yr}$ & $4.92(30-90)$ & 16.40 & Unknown & Unknown & p.G101V/c.1107 + 1G > T \\
\hline
\end{tabular}




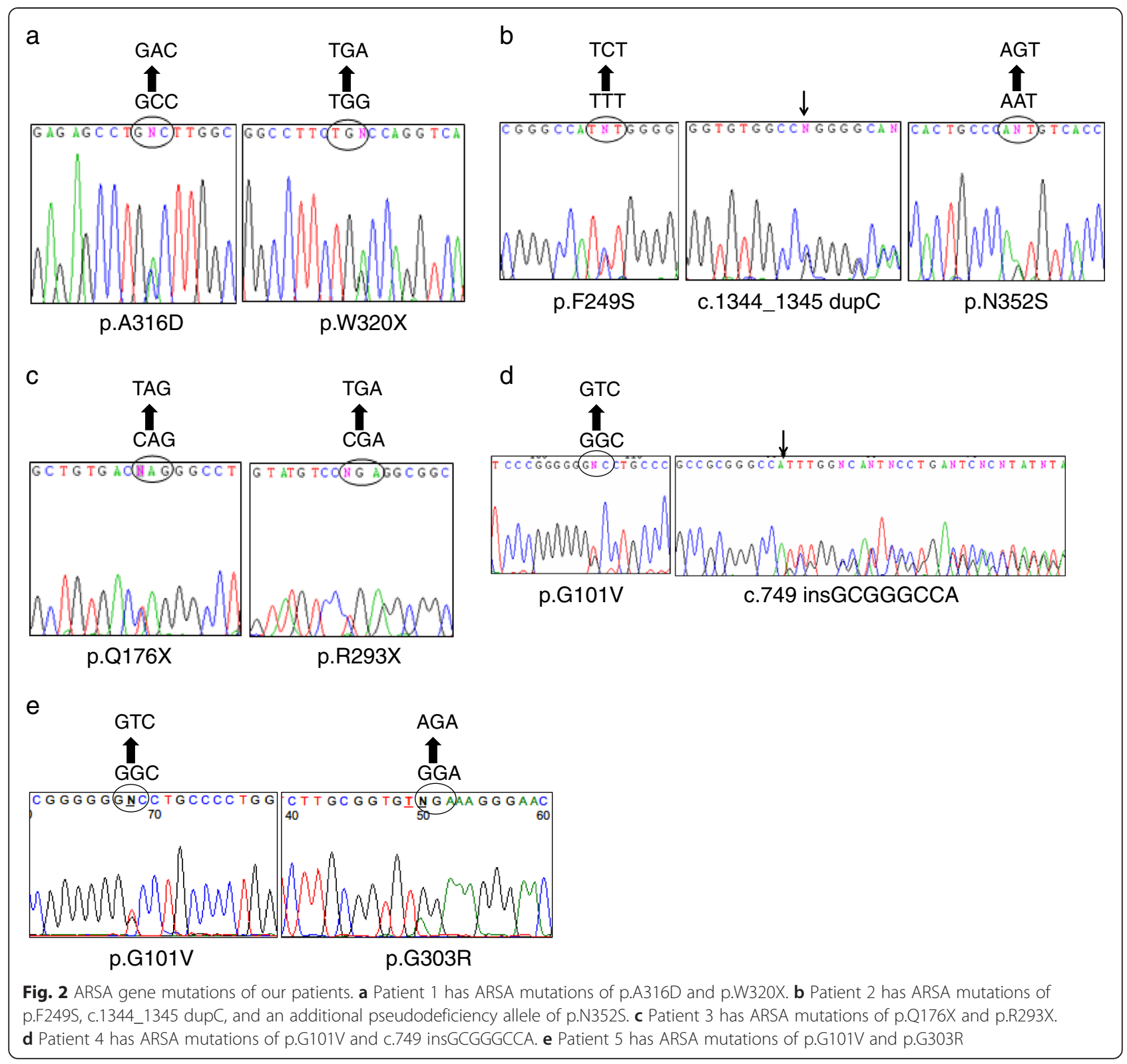

In Asian countries, the number of reported cases with late infantile MLD is limited [13-19]. However, a great diversity of 22 different alleles of ARSA gene mutations was found (Table 2). Five novel mutations of ARSA gene were identified in our patients, including p.A316D, p.G303R, p.Q176X, p.R293X, and c.749 insGCGGGCCA. As the frequency of ARSA mutation alleles in normal alleles and pseudodeficiency alleles was equivalent $[33,34]$, it is possible that patients with late infantile MLD carry both disease-causing and pseudodeficiency alleles. Mutations whose effects might be exaggerated by the reduced synthesis of ARSA due to pseudodeficiency alleles are likely to be seen in late infantile MLD patients carrying both of them [33]. One of our 5 patients, patient 2, carried the ARSA gene mutations p.F249S and c.1344_1345 dupC and the ARSA pseudodeficiency allele p.N352S.

\section{Conclusions}

In conclusion, patients with late infantile MLD exhibit a rapid and devastating clinical course. The initial neurological feature is gait disturbance followed by bed-ridden status within a couple of months to 2 years. Tigroid and leopard skin pattern of dysmyelination on brain MRI together with peripheral demyelination polyneuropathy serve as a clue indicating that evaluation of ARSA activity in leukocytes is warranted. Variable ARSA gene 
mutations could be found in patients with late infantile MLD in Asia, including p.W320X which was found in Taiwan and China, and p.G101V in Taiwan and Korea. An accurate diagnosis of late infantile MLD should be based on a combination of clinical features, biochemical analysis of low residual enzyme activity of ARSA in leukocytes, and pathognomonic ARSA gene mutations, in order to facilitate genetic counseling of family members and prenatal diagnosis.

\section{Abbreviations}

AEP: Auditory evoked potential; ARSA: Arylsulfatase A; CNS: Central nervous system; CSF: Cerebrospinal fluid; DNA: Deoxyribonucleic acid;

EEG: Electroencephalography; MLD: Metachromatic leukodystrophy; MRI: Magnetic resonance imaging; NCV: Nerve conduction velocity; PCR: Polymerase chain reaction; VEP: Visual evoked potential.

\section{Competing interests}

The authors declare that they have no competing interests.

\section{Authors' contributions}

HRL participated in acquisition of data, analysis and interpretation of data, and drafting the manuscript. HFL made great contributions to recruitment of patients and interpretation of data, and she revised the manuscript critically for important intellectual content. CSC participated in the design of the study and recruitment of patients, and he gave final approval of the version. CRT carried out the molecular genetic studies and participated in the sequence alignment. All authors read and approved the final manuscript.

\section{Acknowledgements}

Funding for this study is supported by Taichung Veterans General Hospital.

\section{Author details}

'Department of Pediatrics, Tungs' Taichung Metroharbor Hospital, 699, Taiwan Boulevard Sec. 8, Wuchi, Taichung 435, Taiwan. ${ }^{2}$ Department of Pediatrics, Taichung Veterans General Hospital, 1650, Taiwan Boulevard Sec. 4, Taichung 40705, Taiwan. ${ }^{3}$ School of Medicine, Chung Shan Medical University, 110, Sec. 1, Jianguo N. Rd, Taichung 40201, Taiwan. ${ }^{4}$ Institute of Molecular Biology, National Chung Hsing University, 250, Kuo Kuang Rd, Taichung 402, Taiwan.

Received: 30 June 2015 Accepted: 30 October 2015 Published online: 09 November 2015

\section{References}

1. Patil SA, Maegawa GH. Developing therapeutic approaches for metachromatic leukodystrophy. Drug Des Devel Ther. 2013;7:729-45.

2. Gieselmann V. Metachromatic leukodystrophy: recent research developments. J Child Neurol. 2003;18:591-4

3. Aqarwal A, Shipman PJ. Gallbladder polyposis in metachromatic leukodystrophy. Pediatr Radiol. 2013;43:631-3.

4. Kliegman RM, Stanton BF, St Geme JW, Schor NF, Behrman RE. Neurodegenerative disorders of childhood. In: Kwon JM, editor. Nelson Textbook of Pediatrics. 19th ed. Philadelphia: WB Saunders; 2011. p. 2072.

5. Luzi $P$, Rafi MA, Rao HZ, Wenger DA. Sixteen novel mutations in the arylsulfatase A gene causing metachromatic leukodystrophy. Gene. 2013;530:323-8.

6. Wang RY, Bodamer OA, Watson MS, Wilcox WR. Lysosomal storage diseases: diagnostic confirmation and management of presymptomatic individuals. Genet Med. 2011;13:457-84.

7. Kehrer C, Blumenstock G, Gieselmann V, Krägeloh-Mann I. The natural course of gross motor deterioration in metachromatic leukodystrophy. Dev Med Child Neurol. 2011;53:850-5.

8. Kehrer C, Groeschel S, Kustermann-Kuhn B, Bürger F, Köhler W, Kohlschütter A, et al. Language and cognition in children with metachromatic leukodystrophy: onset and natural course in a nationwide cohort. Orphanet J Rare Dis. 2014;9:18.

9. Boucher AA, Miller W, Shanley R, Ziegler R, Lund T, Raymond G, et al. Long-term outcomes after allogeneic hematopoietic stem cell transplantation for metachromatic leukodystrophy: the largest singleinstitution cohort report. Orphanet J Rare Dis. 2015;10:94.

10. Martin HR, Poe MD, Provenzale JM, Kurtzberg J, Mendizabal A, Escolar ML. Neurodevelopmental outcomes of umbilical cord blood transplantation in metachromatic leukodystrophy. Biol Blood Marrow Transplant. 2013;19:616-24.

11. Biffi A, Montini E, Lorioli L, Cesani M, Fumagalli F, Plati T, et al. Lentiviral hematopoietic stem cell gene therapy benefits metachromatic leukodystrophy. Science. 2013;341:1233158.

12. van Egmond ME, Pouwels PJ, Boelens JJ, Lindemans CA, Barkhof F, Steenwijk MD, et al. Improvement of white matter changes on neuroimaging modalities after stem cell transplant in metachromatic leukodystrophy. JAMA Neurol. 2013;70:779-82.

13. Shukla P, Vasisht S, Srivastava R, Gupta N, Ghosh M, Kumar M, et al. Molecular and structural analysis of metachromatic leukodystrophy patients in Indian population. J Neurol Sci. 2011;301:38-45.

14. Tsuda T, Hasegawa $Y$, Eto $Y$. Two novel mutations in a Japanese patient with the late-infantile form of metachromatic leukodystrophy. Brain Dev. 1996;18:400-3.

15. Kurosawa $\mathrm{K}$, Ida $\mathrm{H}$, Eto $\mathrm{Y}$. Prevalence of arylsulphatase $\mathrm{A}$ mutations in 11 Japanese patients with metachromatic leukodystrophy: Identification of two novel mutations. J Inherit Metab Dis. 1998;21:781-2.

16. Niida Y, Kuroda M, Mitani Y, Yokoi A, Ozaki M. Paternal uniparental isodisomy of chromosome 22 in a patient with metachromatic leukodystrophy. J Hum Genet. 2012;57:687-90.

17. Wang J, Zhang W, Pang H, Bao X, Wu Y, Wu X, et al. ARSA gene mutations in five Chinese metachromatic leukodystrophy patients. Pediatr Neurol. 2007;36:397-401.

18. Yang J, Cao J, Li Y, Zheng H, Li J, Liang Y, et al. Analysis of phenotype and genotype in a family with late infantile metachromatic leukodystrophy. Zhonghua Yi Xue Yi Chuan Xue Za Zhi. 2014;31:615-8.

19. Kang $\mathrm{DH}$, Lee $\mathrm{DH}$, Hong $\mathrm{YH}$, Lee $\mathrm{ST}$, Jeon $\mathrm{BR}$, Lee $\mathrm{YK}$, et al. Identification of a novel splicing mutation in the ARSA gene in a patient with late-infantile form of metachromatic leukodystrophy. Korean J Lab Med. 2010;30:516-20.

20. Mahmood A, Berry J, Wenger DA, Escolar M, Sobeih M, Raymond G, et al. Metachromatic leukodystrophy: a case of triplets with the late infantile variant and a systematic review of the literature. J Child Neurol. 2010;25:572-80.

21. Groeschel S, Kehrer C, Engel C, I Dali C, Bley A, Steinfeld R, et al. Metachromatic leukodystrophy: natural course of cerebral MRI changes in relation to clinical course. J Inherit Metab Dis. 2011;34:1095-102.

22. Groeschel S, í Dali C, Clas P, Böhringer J, Duno M, Krarup C, et al. Cerebral gray and white matter changes and clinical course in metachromatic leukodystrophy. Neurology. 2012;79:1662-70.

23. Martin A, Sevin C, Lazarus C, Bellesme C, Aubourg P, Adamsbaum C. Toward a better understanding of brain lesions during metachromatic leukodystrophy evolution. Am J Neuroradiol. 2012;33:1731-9.

24. Assadi M, Wang DJ, Velazquez-Rodriquez Y, Leone P. Multi-voxel 1H-MRS in metachromatic leukodystrophy. J Cent Nerv Syst Dis. 2013;5:25-30.

25. Bindu PS, Mahadevan A, Taly AB, Christopher R, Gayathri N, Shankar SK Peripheral neuropathy in metachromatic leukodystrophy. A study of 40 cases from south India. J Neurol Neurosurg Psychiatry. 2005;76:1698-701.

26. Cameron CL, Kang PB, Burns TM, Darras BT, Jones Jr HR. Multifocal slowing of nerve conduction in metachromatic leukodystrophy. Muscle Nerve. 2004;29:531-6.

27. Maia Jr AC, da Rocha AJ, da Silva CJ, Rosemberg S. Multiple cranial nerve enhancement: a new MR imaging finding in metachromatic leukodystrophy. Am J Neuroradiol. 2007:28:999.

28. Toldo I, Carollo C, Battistella PA, Laverda AM. Spinal cord and cauda equina MRI findings in metachromatic leukodystrophy: case report. Neuroradiology. 2005:47:572-5.

29. Nandhagopal R, Krishnamoorthy SG. Neurological picture. Tigroid and leopard skin pattern of dysmyelination in metachromatic leucodystrophy. J Neurol Neurosurg Psychiatry. 2006;77:344.

30. Kohlschütter A, Eichler F. Childhood leukodystrophies: a clinical perspective. Expert Rev Neurother. 2011;11:1485-96.

31. Alessandri MG, De Vito G, Fornai F. Increased prevalence of pervasive developmental disorders in children with slight arylsulfatase A deficiency. Brain Dev. 2002;24:688-92.

32. Lorioli L, Cesani M, Regis S, Morena F, Grossi S, Fumagalli F, et al. Critical issues for the proper diagnosis of metachromatic leukodystrophy. Gene. 2014;537:348-51. 
33. Gieselmann V, Fluharty AL, Tønnesen T, Von Figura K. Mutations in the arylsulfatase A pseudodeficiency allele causing metachromatic leukodystrophy. Am J Hum Genet. 1991:49:407-13.

34. Rafi MA, Coppola S, Liu SL, Rao HZ, Wenger DA. Disease-causing mutations in cis with the common arylsulfatase A pseudodeficiency allele compound the difficulties in accurately identifying patients and carriers of metachromatic leukodystrophy. Mol Genet Metab. 2003;79:83-90.

Submit your next manuscript to BioMed Central and take full advantage of:

- Convenient online submission

- Thorough peer review

- No space constraints or color figure charges

- Immediate publication on acceptance

- Inclusion in PubMed, CAS, Scopus and Google Scholar

- Research which is freely available for redistribution 